\title{
To Be or Not to Be... Sepsis? A Daily Challenge in ICU
}

\author{
Adrian Man ${ }^{*}$, Bianca Liana Grigorescu² \\ 1 Department of Microbiology, George Emil Palade University of Medicine, Pharmacy, Sciences and Technology of \\ Targu Mures, Romania \\ 2 Department of Pathophysiology, George Emil Palade University of Medicine, Pharmacy, Sciences and Technology of \\ Targu Mures, Romania
}

Received: 29 January 2020 / Accepted: 31 January 2020

Nowadays, one of the most challenging medical issues is related to high morbidity and mortality in sepsis and septic shock. Despite the medical progress regarding early diagnosis and management, this complex pathology remains a life-threatening condition. During the last decades, many definitions and including criteria were developed both in sepsis and septic shock, principally as many early biomarkers became available. However, many issues still exist regarding this subject.

The clinical definitions of sepsis and systemic inflammatory response syndrome (SIRS) have been refined, but both conditions manifest with similar clinical features [1]. The Third International Consensus Definitions Task Force (Sepsis-3) defined sepsis as "a life-threatening organ dysfunction resulting from a dysregulated host response to infection". Septic shock is "a subset of sepsis in which circulatory, cellular and metabolic alterations are associated with a higher mortality rate than sepsis alone" [2]. These definitions are related to the pathophysiology of sepsis, which are the cornerstones of a better understanding of the underlying mechanisms and disorders that occur [3].

An important concern is related to the early diagnosis and management of sepsis and septic shock developed in ICU patients, admitted for other pathologies. It is well known that the main feature of critical illness is related to the non-infectious systemic inflammatory response to various stressors such as trauma, surgical procedures and other organ dysfunctions secondary to decompensation of an underlying disease. Clinical findings such as fever, changes in heart rate, tachypnea, the need for mechanical ventilation, mental status alterations, hypotension correlated with white blood cell count changes, elevated, lowered and neutrophilia, thrombocytopenia and coagulation abnormalities are parameters used in clinical practice to define SIRS but were also considered as inclusion criteria for sepsis [3]. Moreover, the diagnosis of sepsis became difficult in patients who present comorbidities that mimic sepsis, especially in ICU units.

The second main issue is related to Chronic Critical Illness (CCI) described in patients who have undergone a prolonged length of stay in an ICU. These patients may present with recurrent infections, organ dysfunction, catabolic states and impaired cognitive function, as well as persistent inflammation-immune suppression catabolism syndrome (PICS). The main feature of PICS is ongoing inflammation, leading to immunological dyscrasia [4]. The conditions described above are the main factors that could delay, under or over-diagnose sepsis and septic shock.

Because the primary mechanism of developing sepsis is related to the imbalance of the immune response leading to profound alterations in microcirculation and rapid evolution to multiple organ dysfunction, early diagnosis and treatment are mandatory. In this regard, clinicians should treat sepsis as a medical emergency. The most important change in The Surviving Sepsis Campaign Bundle 2018 is that the 3-hour and 6-hour bundles have been combined in "hour-1 bundle", that highlight the importance of beginning resuscitation and management immediately [5]. This "gold-hour" is vital for beginning aggressive resuscitation as well as for measuring serum lactate levels and obtaining blood cultures. This requires a well-prepared laboratory that can detect the etiological agents of sepsis from blood cultures, which is a requirement for diagnosis. Nowadays, the procedure for obtaining a blood culture is straightforward, and its value is incontestable [6]. When sepsis occurs, bacteria are periodically discharged in the blood system from an infectious starting point, the location and severity of which can vary. Dur- 
ing blood culture, bacteria that are freely flowing in the blood system are multiplied exponentially in a specific liquid culture medium that is included in two different types of bottles; aerobic or anaerobic (one set), with or without antibiotic inhibitor, or pediatric bottles for small volumes of blood [7]. During incubation of the blood culture, bacteria from the harvested blood will start to multiply exponentially, with a rate of around three generations/hour, and the bacterial growth is automatically detected in the incubator. All these make this method highly sensitive and specific, but understandably reliant on the procedures being correctly performed.

An important drawback of the blood culture method is that any bacteria that enters the blood culture flask will grow and induce a positive signal and culture. If these bacteria did not originate from the patient's blood sample but from the commensal flora of the patient's body, or the handler's microflora, or any other external source, it is referred to as blood culture contamination. This makes it difficult for a practitioner to correctly interpret the data in relation to the case. There is a high chance of contaminating blood cultures if the harvesting procedures do not strictly following aseptic rules. Considering that most blood cultures are harvested by venipuncture and skin microflora consists mostly of coagulase-negative staphylococci (CNS), it can be expected that a high rate of CNS positive blood cultures will result if appropriately collecting techniques are not followed [8]. On the other hand, CNS are potent biofilm producers especially on prosthetic devices such as intravascular devices such as catheters, central catheters, prosthesis, chemotherapy chambers and others. These devices can become real sources of bacteremic syndromes, and a positive blood culture should be considered as a real result, with clinical implications.

Considering all of these problems, a CNS result from blood culture should be critically interpreted, and the patient should be checked for the presence of intravascular devices. If present, they should be replaced immediately, and the clinical symptoms should resolve spontaneously without antibiotic therapy. If the intravascular devices are not replaced, antibiotic therapy will not resolve the bacteremic syndrome, as the antibiotic cannot effectively penetrate and act on the bacterial biofilm. Moreover, by administering antibiotherapy, there is a high risk of selecting resistant bacterial strains and aggravate the status of the patient with subsequent infections which are not easily manageable.
Beside CNS, other skin-related microflora contamination can occur with Corynebacterium spp., Micrococcus spp., or anaerobic bacteria such as Cutibacterium spp. (formerly Propionibacterium spp.).

How and why does contamination occur? Contamination occurs mostly because aseptic harvesting procedures have not been respected. Aspiration from catheters is accompanied by a high risk of contamination, as does insufficient antisepsis of the skin, re-palpation of the vein after skin antisepsis, aspiration by syringe and distribution in blood culture bottles.

Apart of the common CNS contamination, there are more alarming situations which can be hard to interpret. Cases of a positive blood culture with multidrug-resistant microbes such as ESBL (extended-spectrum betalactamase-producing) enterobacterieceae, CPE (carbapenemase-producing enterobacterieceae), MRSA (methicillin-resistant Staphylococcus aureus) or VRE (vancomycin-resistant Enterococcus) are some of those in question. These strains are highly prevalent in a hospital environment and indeed can produce a septic state. The sepsis source should be identified, and blood culture results should be correlated with the clinical state, previous and long time hospitalizations, and immune-suppression [9].

Discrepancy in the results should also be considered by the clinician when interpreting the laboratory results. For example, it is uncommon to have sepsis when a non-fastidious, facultatively anaerobic bacteria such as Staphylococcus aureus or Klebsiella spp. is growing in only one blood culture bottle (aerobic or anaerobic). They should grow independently of the environmental conditions.

In cases where only one blood culture bottle (aerobic or anaerobic) is positive, the etiological agent is either obligate aerobic bacteria (e.g. Pseudomonas aeruginosa) or obligate anaerobic bacteria (e.g. Bacteroides spp., anaerobic streptococci, Clostridium spp.). But note that these may also only be contaminants. Alternatively, if two sets of blood cultures harvested from different body sites or from different, but close, timepoints show bacterial growth with different species, which may also suggest contamination. Blood cultures that become positive only after more than 3-4 days of incubation are likely contaminated [10].

Recovery of some bacteria from blood culture such as Staphylococcus aureus, Streptococcus pneumoniae, Streptococcus pyogenes or agalactiae, Enterobacteriace- 
ae, Pseudomonas aeruginosa, Listeria monocytogenes, Neisseria meningitidis, Haemophilus influenzae, Bacteroides fragilis almost always represent a true bacteremia and sepsis [10].

Nevertheless, do not forget that these may also be contaminants. Furthermore, there are problems regarding blood cultures collected from patients in whom broad spectrum antibiotherapy has been initiated. In these cases, the microbiological findings can be impaired because, after the first dose of an appropriate antimicrobial agent, inhibition of bacterial growth can occur in minutes, and the blood culture will remain negative despite the presence of a real pathogen.

Apart from blood cultures, we must highlight the importance of serial measurements of biomarker levels e.g. C-reactive protein, procalcitonin. These two biomarkers possess the capacity to differentiate between infective and non-infective disease [11]. It is not easy to predict sepsis based on individual biomarkers without considering their dynamics and other factors affecting their synthesis. IL- 6 cytokine seems to be a promising biomarker in sepsis since it is involved in triggering and modulating the inflammatory response to microbial injury. Its fast dynamics, with a rapid increase of IL-6 levels in the early stages of sepsis are important features which make it a good biomarker for sepsis; surprisingly it is not routinely used [12].

Indeed, sepsis diagnosis and its management is a daily challenge not only to intensivists but also to all the involved practitioners. Considering this, we strongly support the idea of interdisciplinary collaboration, especially between clinicians and laboratory. The issues highlighted in this paper support the idea that sepsis and septic shock remain a medical emergency requiring rapid diagnosis and therapeutic intervention. These conditions could be over- or under-diagnosed, and sometimes the only response to the question "to be or not to be sepsis" remains the resolution of the disease after broad-spectrum antibiotherapy and vital support measures.

\section{Q CONFLICT OF INTEREST}

None to declare.

\section{REFERENCES}

1. Brodska H, Valenta J, Pelinkova K, Stach Z, Sachl R, Balik M, et al. Diagnostic and prognostic value of presepsin vs. established biomarkers in critically ill patients with sepsis or systemic inflammatory response syndrome. Clin Chem Lab Med. 2018 28;56(4):658-68.

2. Singer $M$, Deutschman CS, Seymour CW, Shankar-Hari $M$, Annane D, Bauer M, et al. The Third International Consensus Definitions for Sepsis and Septic Shock (Sepsis-3). JAMA. 2016 Feb 23;315(8):801-10.

3. Kleinpell RM, Schorr CA, Balk RA. The New Sepsis Definitions: Implications for Critical Care Practitioners. Am J Crit Care Off Publ Am Assoc Crit-Care Nurses. 2016 Sep;25(5):457-64.

4. Mira JC, Gentile LF, Mathias BJ, Efron PA, Brakenridge SC, Mohr AM, et al. Sepsis Pathophysiology, Chronic Critical Illness, and Persistent Inflammation-Immunosuppression and Catabolism Syndrome. Crit Care Med. 2017 Feb;45(2):253-62.

5. Levy MM, Evans LE, Rhodes A. The Surviving Sepsis Campaign Bundle: 2018 update. Intensive Care Med. 2018;44(6):925-8.

6. Dailey PJ, Osborn J, Ashley EA, Baron EJ, Dance DAB, Fusco D, et al. Defining System Requirements for Simplified Blood Culture to Enable Widespread Use in Resource-Limited Settings. Diagn Basel Switz. 2019 Jan 11;9(1).

7. Ombelet $\mathrm{S}$, Barbé B, Affolabi D, Ronat J-B, Lompo P, Lunguya $\mathrm{O}$, et al. Best Practices of Blood Cultures in Low- and MiddleIncome Countries. Front Med [Internet]. 2019 Jun 18 [cited 2020 Jan 29];6. Available from: https://www.ncbi.nlm.nih.gov/ pmc/articles/PMC6591475/

8. He Y, Zhao H, Wei Y, Gan X, Ling Y, Ying Y. Retrospective Analysis of Microbial Colonization Patterns in Central Venous Catheters, 2013-2017. J Healthc Eng [Internet]. 2019 Sep 17 [cited 2020 Jan 29];2019. Available from: https://www.ncbi.nlm.nih.gov/ pmc/articles/PMC6766096/

9. Chang C-J, Wu C-J, Hsu H-C, Wu C-H, Shih F-Y, Wang S-W, et al. Factors Associated with Blood Culture Contamination in the Emergency Department: Critical Illness, End-Stage Renal Disease, and Old Age. PLoS ONE [Internet]. 2015 Oct 8 [cited 2020 Jan 29];10(10). Available from: https://www.ncbi.nlm.nih. gov/pmc/articles/PMC4598129/

10. Hall KK, Lyman JA. Updated Review of Blood Culture Contamination. Clin Microbiol Rev. 2006 Oct;19(4):788-802.

11. Dolin HH, Papadimos TJ, Stepkowski S, Chen X, Pan ZK. A Novel Combination of Biomarkers to Herald the Onset of Sepsis Prior to the Manifestation of Symptoms. Shock Augusta Ga. 2018;49(4):364-70.

12. Georgescu AM, Bănescu C, Badea I, Moldovan V, Huțanu A, Voidăzan $S$, et al. IL-6 gene polymorphisms and sepsis in icu adult romanian patients: a prospective study. Rev Romana Med Lab. 2017 Mar 1;25(1):75-89. 